\title{
Die Folketingswahl in Dänemark vom 15. September 2011: Abwahl einer Regierung ohne klaren Sieg der Opposition
}

\author{
Christoph Arndt
}

Seit der Folketingswahl 2001 wurde Dänemark von einer Koalition der rechtsliberalen Venstre und der Konservativen Volkspartei regiert, die durch die rechtsgerichtete Dänische Volkspartei toleriert wurde. Diese Konstellation sicherte seit 2001 eine dauerhafte parlamentarischer Mehrheit, die nach 2005 auch im Jahre 2007 erneut bestätigt und mitunter um die Liberale Allianz erweitert worden war. ${ }^{1}$ Vor der Folketingswahl im September 2011 wurde das ansonsten durch Minderheitsregierungen und lagerübergreifende parlamentarische Übereinkommen geprägte Land somit ein Jahrzehnt mit einer faktischen parlamentarischen Mehrheit regiert, da die Dänische Volkspartei sich als stabiler und loyaler Partner erwies (so genannte Støtteparti). ${ }^{2}$ Dies führte jedoch zu einer politischen Lagerbildung in einen „blauen“ Block bestehend aus Venstre, den Konservativen, der Dänischen Volkspartei sowie seit 2007 der Liberalen Allianz und einen „roten“ Block aus Sozialdemokraten, Sozialliberalen, Sozialistischer Volkspartei und der Einheitsliste. Aufgrund der Aussagen einiger Spitzenpolitiker, die ein Ende der Blockpolitik unabhängig vom Wahlergebnis ankündigten, war der Ausgang der Folketingswahl 2011 mit Spannung erwartet worden. Die im September erzielte äußerst knappe parlamentarische Mehrheit für eine sozialdemokratisch geführte Regierung deutet aber doch auf eine Fortsetzung der Blockpolitik mangels alternativer Mehrheiten hin.

\section{Die Ausgangslage}

Die Folketingswahl vom 13. November 2007 hatte abermals die 2001 gebildete bürgerliche Regierung des populären Ministerpräsidenten Anders Fogh Rasmussen aus dessen rechtsliberaler Venstre (V), der Konservativen Volkspartei (Det Konservative Folkeparti, K) unterstützt durch die Dänische Volkspartei (Dansk Folkeparti, DF) bestätigt. Ihre knappe parlamentarische Mehrheit wurde im Verlauf der Wahlperiode 2007 bis 2011 durch die Neue Allianz (später Liberale Allianz, LA) als weitere parlamentarische Basis verstärkt.

Die Opposition, bestehend aus Sozialdemokraten (S), Sozialistischer Volkspartei (Socialistisk Folkeparti, SF), der sozialliberalen Radikale Venstre (RV) sowie der linksradikalen Einheitsliste (Enhedslisten, EL), hatte sich im Laufe der Wahlperiode darauf verständigt, im Falle eines Regierungsverlustes der bürgerlichen Parteien die Vorsitzende der Sozialdemokraten Helle Thorning-Schmidt zur neuen Ministerpräsidentin zu wählen. Die Sozialdemokraten und die Sozialistische Volkspartei bildeten spätestens seit 2009 eine prä-elektorale Koalition, wobei beide Parteien für den Fall einer Regierungsübernahme eine Koalition

1 Vgl. Lars Bille, Den Partipolitiske Udvikling 2001 til 2011, in: Økonomi \& Politik, 84. Jg. (2011), H. 3, S. 3 - 18.

2 Zur Funktionsweise des dänischen Parlamentarismus unter Minderheitsregierungen und dem Übergang zur Blockpolitik Christoffer Green-Pedersen / Lisbeth Hoffmann Thomsen, Bloc Politics vs. Broad Cooperation? The Functioning of Danish Minority Parliamentarism, in: Journal of Legislative Studies, 11. Jg. (2005), H. 2, S. $153-169$. 
garantierten. ${ }^{3} S$ und SF demonstrierten zudem eine teilweise Akzeptanz der restriktiven Ausländerpolitik der bürgerlichen Regierung und der Dänischen Volkspartei - auch um diesem Thema die politische Brisanz zu nehmen und wirtschafts- und sozialpolitische Themen in den Vordergrund zu rücken. Dieses Vorgehen wurde von den anderen beiden Oppositionsparteien, der Radikalen Venstre und der Einheitsliste, kritisiert, die eine liberale Zuwanderungspolitik befürworteten.

Im Verlauf der Wahlperiode verlor die bürgerliche Regierung mitsamt der Dänischen Volkspartei ihre knappe parlamentarische Mehrheit infolge von Parteiwechseln und Fraktionsaustritten einzelner Abgeordneter. V, K und DF waren nun neben der Liberalen Allianz somit zum Teil auf Unterstützung fraktionsloser Abgeordneter sowie des Christdemokraten Per Ørum Jorgensen angewiesen, der 2010 nach einer Trunkenheitsfahrt von den Konservativen zu den bisher nicht im Folketing vertretenen Christdemokraten wechselte. ${ }^{4}$ Zudem wurde Ministerpräsident Anders Fogh Rasmussen zum NATO-Generalsekretär berufen und am 5. April 2009 durch den bisherigen Finanzminister Lars Løkke Rasmussen ersetzt.

Angesichts der ökonomischen Auswirkungen der Finanzkrise und den schlechten Umfragewerten vollzog Løkke Rasmussen am 23. Februar 2010 eine umfassende Kabinettsumbildung. Diese sollte einen Kurswechsel hin zu einem bürgerlicheren Profil, als es unter dem moderateren Vorgänger Fogh Rasmussen der Fall war, markieren und einige weitreichende ökonomische Reformen auf den Weg bringen. Am 19. Mai 2010 präsentierten die Vorsitzenden der Regierungsparteien Lars Løkke Rasmussen (Venstre) und Lene Espersen (Konservative) ein Sparprogramm, das Einsparungen in Höhe von 24 Milliarden dänischen Kronen vorsah. ${ }^{5}$ Es halbierte unter anderem die Bezugsdauer des Arbeitslosengeldes von vier auf zwei Jahre und sah ein Nullwachstum vieler Transferleistungen und der Beschäftigung im öffentlichen Sektor vor. ${ }^{6}$ Die Radikale Venstre befürwortete einige Sparmaßnahmen, so dass erste Spannungen zwischen den Oppositionsparteien im Bereich der Wirtschaftspolitik sichtbar wurden.

Wirtschafts- und finanzpolitische Themen dominierten in der Folge die politische Agenda, und in seiner Neujahrsansprache 2011 kündigte Ministerpräsident Rasmussen weitere Sparmaßnahmen an, insbesondere eine Reform der Frühverrentung (Efterløn). Inzwischen wurde Lene Espersen nach mehrjährigen internen Auseinandersetzungen am 14. Januar 2011 durch Lars Barfoed an der Spitze der Konservativen abgelöst. ${ }^{7}$

Im April 2011 beschloss die Regierung ein weiteres Sparprogramm im Umfang von 47 Milliarden Kronen, dass insbesondere eine Reform der gesetzlichen Rente (Folkepension) sowie der Frührente (Efterløn) umfasste. Im Mai 2011 einigten sich Regierung, Dänische Volkspartei und die Radikale Venstre unter heftigem Protest der anderen drei Oppositionsparteien auf eine Reform, die eine Verkürzung der Bezugsdauer des Efterløns von fünf auf drei Jahre, eine Erhöhung des gesetzlichen Renteneintrittsalters von 65 auf 67 Jahre sowie die Erhöhung des Eintrittsalters für den Efterløn auf 64 Jahre vorsah. Eine Konzession der

3 Siehe hierzu Lars Bille, a.a.O. (Fn. 1), S. $13 \mathrm{ff}$.

4 Vgl. Marie Sahl / Marie Hjortdal, VKO har mistet sit Flertal, in: Politikken vom 20. Mai 2010.

5 Vgl. Mads Brandstrup, Regeringen: Nu skal vi alle sammen bidrage, in: Politikken vom 20. Mai 2010.

6 Siehe hierzu die Regierungserklärung GenopretningsPakken. Danmark ud af Krisen - Regningen betalt, Finansministeriet, Kopenhagen Mai 2010.

7 Zuvor hatte es bereits im September 2008 einen Wechsel gegeben, als Lene Espersen den Vorsitz der Konservativen von Bendt Bendtsen übernahm. 
Regierung an die Dänische Volkspartei hierbei war die Verstärkung der Kontrolle an den Grenzen zu Deutschland und Schweden.

Spätestens seit dieser Übereinkunft kam die Frage nach einer Neuwahl des Folketings auf, insbesondere als Möglichkeit, den Reform- und Sparkurs der Regierung bestätigt zu bekommen. Eine Wahl zum Folketing muss laut Dänischer Verfassung spätestens vier Jahre nach der letzten Wahl vom Ministerpräsidenten ausgeschrieben werden, so dass der letztmögliche Termin der 13. November 2011 war. Ministerpräsident Løkke Rasmussen kündigte am 26. August die Wahl für den 15. September an, nachdem Verhandlungen über ein Konjunkturprogramm und die Immobilienwertsteuer zwischen Regierung und Dänischer Volkspartei zu keinem Ergebnis gekommen waren. ${ }^{8}$

\section{Wahlkampf und Themen}

Die Ausgangslage für eine Wiederwahl Løkke Rasmussens zum Ministerpräsidenten war schwierig, da die beiden Regierungsparteien Venstre und Konservative sowie ihre parlamentarische Basis aus Dänischer Volkspartei und Liberaler Allianz seit mehreren Monaten ohne Mehrheit in den Meinungsumfragen waren. Die Sozialdemokraten hatten oft eine Mehrheit zusammen mit den Sozialisten und den Sozialliberalen ohne die Einheitsliste, was eine mögliche Regierungsübernahme erleichtert hätte.

Die Themen des nur drei Wochen andauernden Wahlkampfes setzten die politische Auseinandersetzung der Jahre 2010 und 2011 unter dem Eindruck der Finanzkrise fort. Der Wahlkampf wurde primär von wirtschafts- und sozialpolitischen Themen geprägt und nicht mehr wie in den vorherigen drei Wahlkämpfen durch das Thema Einwanderung, was der zuwanderungsskeptischen Dänischen Volkspartei zugute kam. ${ }^{9}$

An der Spitze stand dabei die Auseinandersetzung um Dänemarks zukünftige Wirtschaftspolitik und die finanzielle Nachhaltigkeit politischer Entscheidungen. Venstre setzte hierbei auf nachhaltiges Wirtschaftswachstum, betonte die finanzpolitische Verantwortung der Partei und warnte vor einem Ansteigen der Neuverschuldung und der Steuern unter einer zukünftigen sozialdemokratisch geführten Regierung. ${ }^{10}$ Zusammen mit den Konservativen und der Dänischen Volkspartei sagte Venstre zu, an der Rentenreform des Frühjahres 2011 festzuhalten, was auch von der oppositionellen Radikalen Venstre bekräftigt wurde. Die Sozialdemokraten im Verbund mit der Sozialistischen Volkspartei versprachen dagegen eine Rücknahme der Reform bei entsprechender parlamentarischer Mehrheit nach der Wahl. ${ }^{11}$ Sozialdemokraten und Sozialisten präsentierten bereits zuvor ein eigenes Programm zur Sicherung von Dänemarks ökonomischer Zukunft, das unter anderem vorsah, geplante öffentliche Investitionen sowie eine längere Wochenarbeitszeit vorzuziehen. ${ }^{12}$ Dies

8 Vgl. Kristian Klarskov / Morten Skarbak / Mads Brandstrup / Martin Kaae, Lidt før Klokken Ni var Lars og Lars færdige med at slås om Datoen, in: Politiken vom 27. August 2011.

9 Siehe hierzu Valgkampens vigtigste Temaer, in: Berlingske Tidende vom 27. August 2011; Økonomien bliver det store Tema, in: Midtjyllands Avis vom 27. August 2011.

10 Vgl. Venstre, Valggrundlag Folketingsvalg den 15. September 2011, Wahlprogramm 2011.

11 Vgl. Socialdemokraterne / Socialistisk Folkeparti, Sammen om Danmark, Gemeinsames Wahlprogramm 2011; Christine Cordsen / Dorte Ipsen Boddum, Thorning vil rulle Reform tilbage, in: Jyllands-Posten vom 13. April 2011.

12 Vgl. Socialdemokraterne / Socialistisk Folkeparti, Fair Løsning 2020 - Sammen om Danmark, Gemeinsames Wirtschaftspolitisches Programm 2010. 
wurde von den Parteien des blauen Blocks als nicht finanzierbar kritisiert, da es expansive Finanzpolitik andeutete sowie nicht gegenfinanzierte Erhöhungen von Ausgaben und Sozialleistungen enthielte.

Die Dänische Volkspartei betonte im Wahlkampf die Notwendigkeit des Festhaltens an einer restriktiven Zuwanderungspolitik und kritisierte insbesondere die Radikale Venstre und die Einheitsliste für deren zu liberalen Kurs. Dieser sei schädlich für Dänemark, da Zuwanderer ohne realistische Integrationschancen in das Land kommen würden. ${ }^{13}$ Des Weiteren wurden stärkere Grenzkontrollen und die Eindämmung der Kriminalität als Ziele der Partei genannt. Sie setzte auf die Fortsetzung einer bürgerlichen Regierung unter Løkke Rasmussen mit der DF als parlamentarischer Basis.

Zuwanderung kam erst spät im Wahlkampf auf die Agenda, war jedoch nicht Hauptthema. Im Wesentlichen drehte sich die Auseinandersetzung um die Beibehaltung der sogenannten 24-Jahre-Regelung, die unter der bürgerlichen Regierung im Jahr 2002 implementiert worden war, um Familiennachzug zu regeln und Zwangsheiraten zu verhindern. Im linken Lager ist diese jedoch umstritten, so dass RV und EL ihre Abschaffung propagierten, während die Sozialdemokraten sie beibehalten wollen. ${ }^{14}$ Die Position von RV und EL wurden von der DF aufgegriffen und scharf kritisiert.

Mit Beginn des Wahlkampfs sorgte zudem der ansonsten als farblos empfundene Spitzenkandidat und neue Vorsitzende der Konservativen Lars Barfoed für Aufmerksamkeit. Zusammen mit der Spitzenkandidatin der Radikalen Venstre Margrethe Vestager schlug er vor, die Blockpolitik unabhängig vom Ausgang der anstehenden Wahl zu beenden und zu lagerübergreifender Kooperationspolitik zurückzukehren. ${ }^{15}$ Dies wurde von einigen politischen Kommentatoren und der Dänischen Volkspartei als Eigentor interpretiert, weil so unzufriedene bürgerliche Wähler animiert werden könnten, für die Radikale Venstre zu stimmen. Diese Wähler hätten nicht mehr zwangsläufig die Politik einer roten Regierung zu befürchten, könnten somit zu den Sozialliberalen wechseln und die bürgerliche Regierung zu Fall bringen. Des Weiteren würde es den Sozialliberalen so ermöglicht, ihre traditionelle Position als pivotale Partei wieder einzunehmen, da diese sich zum Beispiel bei der Rentenreform ebenfalls als Partei der wirtschafts- und finanzpolitischen Vernunft präsentierte, jedoch die restriktive Zuwanderungspolitik der bürgerlichen Regierung ablehnte. ${ }^{16}$ Seit den 1960er Jahren und bis zur Wahl 2001 waren die Sozialliberalen Dänemarks pivotale Partei, da in aller Regel weder reine mitte-links noch mitte-rechts Regierungen eine parlamentarische Mehrheit erringen konnten und jeweils die Mandate der Sozialliberalen eine Mehrheit garantierten. Somit konnten diese zwischen sozialdemokratischen und bürgerlichen Regierungen wählen und viele ihrer eigenen Positionen entweder als Koalitionspartner oder durch Tolerierung von Minderheitsregierungen durchsetzen. ${ }^{17}$

Ziel der drei im Parlament vertretenen Kleinparteien Liberale Allianz, Einheitsliste und Christdemokraten war es, über die Zweiprozentsperrklausel zu kommen. Alsbald zeichnete

13 Vgl. Pia Kjersgaard, Valget er klart, in: Jydske Vestkysten vom 12. September.

14 Vgl. Mette Østergaard, Historisk opbrud i rød blok, in: Politiken vom 9. September 2011.

15 Vgl. Barfoed og Vestager i Alliance, in: Fyens Stiftstidende vom 28. August 2011.

16 Siehe hierzu Rikke Struck Westersø / James Kristoffer Miles, Margrethes Mesterværk, in: B.T. vom 29. August 2011; Mette Østergaard, Midten i Dansk Politik er igen aktiveret, in: Politiken vom 29. August 2011.

17 Hierzu Asbjørn Skaveland, Modelling Government Formation in Denmark and Beyond, in: Party Politics, 15. Jg. (2009), H. 6, S. 715 - 735. 
sich jedoch ab, dass dies der Liberalen Allianz mit ihrem klaren marktliberalen Profil sowie der linken Einheitsliste mit ihrer populären und telegenen Spitzenkandidatin Johanne Schmidt Nielsen gelingen würde, die Christdemokraten jedoch chancenlos waren und nur auf ein einziges Wahlkreismandat hoffen konnten. ${ }^{18}$

\section{Das Wablergebnis}

Die Beteilung an Wahlen zum Folketing ist traditionell hoch und gehört zu den höchsten in westlichen Demokratien. Im September 2011 wurde mit 87,7 Prozent die höchste Wahlbeteiligung seit 1984 erreicht. Der Ausgang der Wahl bescherte den Oppositionsparteien zwar einen knappen Sieg, jedoch eine schwierige parlamentarische Situation für eine sozialdemokratisch geführte Regierung. ${ }^{19}$ Diese ist zukünftig sowohl auf die Stimmen der Einheitsliste sowie im Bedarfsfall auf die so genannten Nordatlantischen Mandate angewiesen: Das Folketing hat 179 Sitze, von denen je zwei Abgeordnete auf den Färöern und Grönland gewählt werden. ${ }^{20}$ Obwohl diese vier Abgeordneten den Parteien auf den Färöern und Grönland zugeordnet werden, können sie bei knappen parlamentarischen Verhältnissen den Ausschlag geben, insofern sich deren Abgeordnete mit einer dänischen Partei assoziieren. ${ }^{21}$

Ohne Einberechnung der vier nordatlantischen Abgeordneten erreichte der rote Block 89 Mandate, denen 86 Mandate für den blauen Block gegenüberstehen. Als Ergebnis der Wahl im Nordatlantik werden drei Sitze dem roten Block und einer dem blauen Block zugerechnet. Diese Konstellation erlaubt somit bei parlamentarischen Abstimmungen nur zwei Abweichler, sofern die drei „roten“ nordatlantischen Abgeordneten die Regierung stützen. Allerdings gibt es eine informelle Übereinkunft, dass keine Regierung durch das Abstimmungsverhalten der nordatlantischen Abgeordneten gestürzt werden soll.

Der Wahlausgang war somit wesentlich knapper als von den Meinungsumfragen in den Wochen zuvor angedeutet und brachte einige so nicht prognostizierte Überraschungen (vgl. Tabelle 1). Venstre konnte ihre Position als stärkste dänische Partei überraschend verteidigen und gewann ein Mandat hinzu. Somit sind die Rechtsliberalen seit über einem Jahrzehnt die größte Partei im traditionell sozialdemokratisch geprägten Dänemark. Die

18 Um Mandate im Folketing zu bekommen, muss eine Partei mindestens zwei Prozent der abgegebenen Stimmen erhalten haben oder mindestens ein Wahlkreismandat in einem der zehn Mehrpersonenwahlkreise gewonnen haben.

19 Vgl. Christine Cordsen, En skrøbelig Sejr til Thorning, in: Jyllands-Posten vom 16. September 2011.

20 Wahlen zum 175 Mitglieder umfassenden Folketing werden nach Verhältniswahlrecht mit Mehrpersonenwahlkreisen abgehalten. Die Sitzzuteilung erfolgt seit 2007 nach dem d'Hondt-Verfahren, wobei ein zusätzlicher regionaler Verteilungsschlüssel angewendet wird. Um Mandate im Folketing zu bekommen, muss eine Partei mindestens zwei Prozent der abgegebenen Stimmen erhalten haben oder mindestens ein Wahlkreismandat in einem der zehn Mehrpersonenwahlkreise gewonnen haben. Hierbei ergeben sich 135 so genannte Kredsmandater (in etwa: Wahlkreismandate), die durch 40 Tillægsmandater (Zusatzmandate) ergänzt werden. Dieses Verfahren zielt darauf ab, eine möglichst strikte Proportionalität zu sichern, da kleinere Parteien in der Regel kaum Aussichten auf Kredsmandater haben und ihre Repräsentation über die Zusatzmandate gesichert wird. Wähler haben eine Stimme und können entweder eine Partei direkt wählen oder einen Kandidaten einer Partei.

21 Zur Rolle der Nordatlantischen Mandate bei der Regierungsbildung Asbjørn Skjaveland, Government Formation in Denmark 1953-1998, Aarhus 2003, S. 114 - 124. 


\begin{tabular}{|c|c|c|c|c|c|}
\hline & \multicolumn{3}{|c|}{$\begin{array}{c}\text { Parteienstimmen } \\
\text { und Kandidatenstimmen }\end{array}$} & \multicolumn{2}{|c|}{$\begin{array}{l}\text { Veränderungen } \\
\text { zur Wahl } 2007\end{array}$} \\
\hline & absolut & Prozent & Sitze & $\begin{array}{c}\text { Prozent- } \\
\text { punkte }\end{array}$ & Sitze \\
\hline Wahlberechtigte & 4.709 .910 & 100 & \multirow[t]{4}{*}{$175^{\mathrm{a}}$} & \multirow{4}{*}{$+1,1$} & \\
\hline Wahlbeteiligung & 3.579 .675 & 87,7 & & & \\
\hline Ungültig & 34.307 & 1,0 & & & \\
\hline Gültig & 3.545 .368 & $100^{\mathrm{b}}$ & & & \\
\hline Venstre (V) & 947.725 & 26,7 & 47 & $+0,5$ & +1 \\
\hline Sozialdemokraten $(S)$ & 879.615 & 24,8 & 44 & $-0,7$ & -1 \\
\hline Dänische Volkspartei (DF) & 436.726 & 12,3 & 22 & $-1,6$ & -3 \\
\hline Sozialistische Volkspartei (SF) & 326.192 & 9,2 & 16 & $-3,8$ & -7 \\
\hline Konservative Volkspartei (K) & 175.047 & 4,9 & 8 & $-5,5$ & -10 \\
\hline Radikale Venstre (RV) & 336.698 & 9,5 & 17 & $+4,4$ & +8 \\
\hline Liberale Allianz(LA)c & 176.585 & 5,0 & 9 & $+2,2$ & +4 \\
\hline Einheitsliste (EL) & 236.860 & 6,7 & 12 & $+4,5$ & +8 \\
\hline Christdemokraten (KD) & 28.070 & 0,8 & $0^{d}$ & $-0,1$ & \\
\hline Einzelbewerber & 1.850 & 0,1 & & $+0,1$ & \\
\hline \multicolumn{6}{|c|}{$\begin{array}{l}\text { a Anzahl ohne die vier nordatlantischen Mandate. } \\
\text { b Die Angabe } 100 \text { Prozent bezieht sich auf die Anzahl der gültigen Stimmen. } \\
\text { c } 2007 \text { als Neue Allianz (Ny Alliance) angetreten. } \\
\text { d Ein Mandat seit } 2010 \text { durch Übertritt des Abgeordneten Per Ørum Jørgensen von Konservativen zu } \\
\text { Christdemokraten. } \\
\text { Quelle: Danmarks Statistik, Endgültiges Wahlergebnis. }\end{array}$} \\
\hline
\end{tabular}

Sozialdemokraten verloren einen Sitz und blieben sogar unter dem Resultat von 2007, so dass diese Wahl ihr schlechtestes Ergebnis seit 108 Jahren markiert. Damit verharren die Sozialdemokraten trotz einer Dekade in der Opposition bei der etwa 25 Prozent seit dem Regierungsverlust 2001.22 Die Dänische Volkspartei konnte trotz eines Stimmenrückgangs um 1,6 Prozentpunkte - einhergehend mit dem Verlust dreier Mandate - ihre Position als drittstärkste Kraft konsolidieren. Die Sozialistische Volkspartei war trotz guter Umfragewerte in den Monaten zuvor ein großer Verlierer der Wahl, da sie deutlich an Stimmen und Sitzen verlor. Eine Ablösung der DF als drittstärkste Partei misslang, und am Ende wurde die SF sogar noch von den Sozialliberalen überholt, nachdem sie zum Teil nur noch als Anhängsel der Sozialdemokraten wahrgenommen wurde. ${ }^{23}$ Die Radikale Venstre konnte die Anzahl ihrer Sitze fast verdoppeln und erreichte mit 9,5 Prozent und 17 Mandaten ihr bestes Ergebnis seit 1973.

22 Vgl. Jimmy van der Brugge / Henning Voss, Årsager til de socialistiske Partiers Tilbagegang i Perioden 1990-2005, in: Jorgen Goul Andersen / Johannes Andersen / Ole Borre / Kasper Møller Hansen I Hans Jørgen Nielsen (Hrsg.), Det nye politiske Landskab. Folketingsvalget 2005 i Perspektiv, Aarhus 2007, S. 127 - 152; Christoph Arndt, Denmark, in: ders., The Electoral Consequences of Third Way Reforms: Social Democracy's Transformation and Its Political Costs, Aarhus 2011, S. $141-166$.

23 Vgl. Martin Kaae / Kristian Klarskov, Presset Søvndal udstiller forskellene på S og SF, in: Politikken vom 1. September 2011; Pres på Villy, in: Jyllands-Posten vom 7. September 2011. 
Ein Desaster erlebten die Konservativen, deren Mandats- und Stimmenzahl mehr als halbiert wurde. Das Ergebnis von 4,9 Prozent stellt das schlechteste Wahlresultat in der 96-jährigen Geschichte der Partei dar, die in den 1980ern noch die zweitstärkste Kraft unter Ministerpräsident Paul Schlüter war. Als Folge sind die Konservativen die kleinste der im Folketing vertretenen Parteien, da sie unerwartet von Einheitsliste und Liberaler Allianz überholt wurden. Zudem sind ihre acht Mandate nicht ausreichend, um die angekündigte lagerübergreifende Zusammenarbeit mit den Radikalen zu realisieren, da eine mögliche Kooperation aus S, SF, RV und K über keine parlamentarische Mehrheit verfügt.

Die aus dem Zusammenschluss mehrerer kommunistischer und linkssozialistischer Listen im Jahre 1990 gebildete Einheitsliste erreichte das beste Resultat ihrer Geschichte und konnte ihren Stimmen- und Sitzanteil verdreifachen. Somit ist es nicht möglich, eine Mehrheit des roten Blockes ohne sie zu bilden. Auch die Liberale Allianz verbuchte ein sensationelles Ergebnis. Sie hatte seit ihrem erstmaligen Einzug in das Folketing 2007 noch oft bei 0,0 Prozent in den Umfragen gelegen und konnte erst durch ihre Positionierung als klare marktliberale Partei durch ihren Spitzenkandidaten Anders Samuelson wieder Zuspruch für sich gewinnen.

Die Christdemokraten scheiterten dagegen wie bereits bei den Wahlen 2005 und 2007 an der die Zweiprozenthürde, und ihr Spitzenkandidat Per Ørum Jorgensen erzielte ebenfalls nicht genug Stimmen im Wahlkreis Westjütland, um das erhoffte Wahlkreismandat und somit parlamentarische Repräsentation zu erreichen. Das abermalige Scheitern dürfte wohl das endgültige politische Aus der Christdemokraten einläuten.

\section{Wählerwanderung und Wablmotive}

Die Niederlage der bürgerlichen Regierung wurde im Wesentlichen auf die Gewinne der Sozialliberalen zurückgeführt. Die Analyse der Wählerwanderungen zeigt, dass die Radikalen Stimmen von nahezu allen Parteien hinzugewannen und dabei Wähler vom blauen in den roten Block wechselten (vgl. Tabelle 2). ${ }^{24}$ Insbesondere männliche Wähler wandten sich von den Rechtsliberalen, der Liberalen Allianz und den Konservativen ab und votieren für die Radikale Venstre, was wesentlich zur wenn auch knappen Änderung der Mehrheitsverhältnisse beitrug. ${ }^{25}$ Die Sozialliberalen konnten während des Wahlkampfes auch bei hochgebildeten Wählern Zugewinne erzielen. ${ }^{26}$ Diese Wähler unterstützen mehrheitlich das Eintreten der RV für die Rentenform des Frühjahres, lehnen jedoch die restriktive Zuwanderungspolitik der bisherigen bürgerlichen Regierung ab.

Ein weiterer Grund waren die Stimmenverluste der Dänischen Volkspartei an die Sozialdemokraten. Hierbei dürfte es sich um einen Teil des Wählersegmentes handeln, das 2001 aufgrund der Sozialstaatsreformen und der als zu liberal empfundenen Zuwanderungspolitik der Sozialdemokraten zur Dänischen Volkspartei und Venstre gewechselt war. ${ }^{27}$ Die Beteiligung der DF an der Efterlønsreform wurde von Wahlforschern als wesentlicher Grund für

24 Vgl. Søren Risbjerg Thomsen, De højt uddannede sikrede rød sejr, http://www.altinget.dk/christiansborg/artikel/de-hoejt-uddannede-sikrede-roed-sejr (Abruf am 8. März 2012).

25 Vgl. die Wählerwanderungen nach Geschlecht in Berlingske Tidende - Politiko, http:// www.b.dk/politiko/saadan-har-vaelgerne-skiftet-parti (Abruf am 7. Oktober 2011).

26 Vgl. Søren Risbjerg Thomsen, a.a.O. (Fn. 24).

27 Vgl. Christoph Arndt, a.a.O. (Fn. 20). 


\begin{tabular}{|c|c|c|c|c|c|c|c|c|c|c|}
\hline \multicolumn{11}{|c|}{$\begin{array}{l}\text { Tabelle 2: Wählerwanderungen zwischen Folketingswablen } 2007 \text { und } 2011 \\
\text { (Prozentwerte, Zeilenprozent) }\end{array}$} \\
\hline & \multicolumn{10}{|c|}{ Wahl 2011} \\
\hline & V & $S$ & DF & SF & K & RV & LA & EL & $\mathrm{KD}$ & $\begin{array}{l}\text { Nicht- } \\
\text { wähler }\end{array}$ \\
\hline $\mathrm{V}$ & 72,9 & 2,2 & 5,6 & 0,7 & 1,8 & 2,7 & 5,4 & 0,2 & 0,4 & 7,9 \\
\hline S & 1,6 & 71,6 & 1,5 & 6,0 & 2,5 & 5,9 & 1,2 & 2,9 & 2,5 & 9,0 \\
\hline $\mathrm{DF}$ & 9,0 & $6,8^{\mathrm{b}}$ & 67,1 & 1,8 & 0,4 & 0,8 & 2,0 & 1,5 & 0,0 & 10,8 \\
\hline SF & 2,9 & 13,4 & 2,6 & 44,8 & 0,0 & 6,9 & 0,3 & 20,6 & 0,2 & 8,2 \\
\hline$\stackrel{\Omega}{\stackrel{\sigma}{\circ}} \mathrm{K}$ & 28,7 & 2,6 & 3,4 & 0,6 & 35,6 & 5,9 & 14,6 & 0,4 & 0,6 & 7,3 \\
\hline$\stackrel{\mathrm{RV}}{\sim}$ & 6,1 & 13,1 & 0,0 & 5,3 & 0,8 & 62,3 & 3,3 & 4,1 & 0,0 & 4,9 \\
\hline 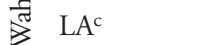 & 8,2 & 6,0 & 3,0 & 3,7 & 16,4 & 25,4 & 23,9 & 3,0 & 0,7 & 10,4 \\
\hline $\mathrm{EL}$ & 0,0 & 4,9 & 1,0 & 5,8 & 0,0 & 3,9 & 1,0 & 82,5 & 0,0 & 1,0 \\
\hline $\mathrm{KD}$ & 4,9 & 7,3 & 2,4 & 7,3 & 0,0 & 17,1 & 0,0 & 2,4 & 51,2 & 9,8 \\
\hline Nichtwähler & 11,4 & 16,1 & 4,8 & 3,5 & 2,1 & 7,5 & 2,5 & 3,7 & 0,8 & 47,9 \\
\hline Erstwähler & 21,4 & 11,8 & 3,9 & 11,4 & 5,0 & 14,3 & 7,5 & 9,6 & 0,4 & 15,0 \\
\hline \multicolumn{11}{|c|}{$\begin{array}{l}\text { a Prozentpunkte beziehen sich auf die Wahlentscheidung } 2011 \text { aufgeschlüsselt nach der Wahlentschei- } \\
\text { dung von } 2007 \text {. } \\
\text { b Entscheidende blockübergreifende Wanderungen fett. } \\
\text { c } 2007 \text { als Neue Allianz angetreten. } \\
\text { Quelle: Eigene Berechnungen basierend auf der Wahlanalyse von Søren Risbjerg Thomsen, Institut for } \\
\text { Statskundskab, Aarhus Universitet } 2011 \text {. }\end{array}$} \\
\hline
\end{tabular}

die Wanderung von DF-Wählern zu den Sozialdemokraten genannt. ${ }^{28}$ Allerdings verloren die Sozialdemokraten und auch Sozialisten während des Wahlkampfes ihrerseits an Zustimmung, da sich Zweifel über die Finanzierbarkeit ihrer Wahlkampfversprechen ausbreiteten und eine geplante PKW-Maut für Kopenhagen potentielle Wähler verschreckte.

Innerhalb des bürgerlichen Lagers fand zudem eine starke Wählerwanderung von den Konservativen zur Venstre und zur Liberalen Allianz statt. Das Desaster der Konservativen zeigte sich insbesondere in ihren Hochburgen nördlich von Kopenhagen, wo sie deutliche Stimmenverluste und ihre Konkurrenten von V, LA, aber auch RV Gewinne verzeichnen konnten. ${ }^{29}$ Ein weiterer Grund für das schwache Abschneiden der Konservativen dürfte das weitgehend blasse Auftreten ihres Spitzenkandidaten Lars Barfoed gewesen sein. Dieser konnte, abgesehen von der angedachten lagerübergreifenden Kooperation mit den Sozialliberalen, keine Akzente oder Themenschwerpunkte im Wahlkampf setzen. Dem Spitzenkandidaten der Venstre und bisherigen Ministerpräsidenten Lars Løkke Rasmussen gelang es während des Wahlkampfes die befürchteten Verluste für seine Partei in einen leichten $\mathrm{Zu}$ gewinn am Wahlabend umzuwandeln. Er verbuchte mit mehr als 56.000 persönlichen Stimmen auch das beste Ergebnis aller angetretenen Kandidaten. ${ }^{30}$

28 Vgl. Rasmus Bøttcher Christensen, Valgforsker: DF er lige så skyldig som K i Løkkes nederlag, http://politiken.dk/politik/ECE1398156/valgforsker-df-er-lige-saa-skyldig-som-k-i-loekkes-nederlag (Abruf am 6. Oktober 2011).

29 Vgl. die Wahlkreisresultate von Danmarks Statistik, http://www.dst.dk/valg/Valg1204271/ valgopg/valgopg.htm (Abruf am 6. Oktober 2011).

30 Siehe hierzu die persönlichen Stimmen der besten zehn Kandidaten, in: Politiken vom 17. September 2011. 
Die Einheitsliste gewann hauptsächlich Stimmen von den Sozialdemokraten und den Sozialisten. Sonst um die Zweiprozentsperrklausel ringend, hatte sie diesmal mit ihrer Spitzenkandidatin Johanne Schmidt Nielsen ein populäres und telegenes Zugpferd im Wahlkampf. ${ }^{31}$ Jedoch wurde die Partei aufgrund vieler utopischer und nicht finanzierbarer Positionen von den auf Schmidt Nielsen fokussierten Medien und den anderen Parteien im Wahlkampf nicht immer ernst genommen und blieb so auch häufig frei von Kritik verschont. ${ }^{32}$ Weitere Analysen des Wahlverhaltens nach Alter und Region ergaben, dass das Alter im Vergleich zur Folketingswahl 2007 keine ausschlaggebenden Effekte hatte. ${ }^{33}$ Lediglich die Liberale Allianz konnte zunehmend bei Wählern unter 40 Jahren punkten, während sie bei älteren Wählern deutlich schwächer abschnitt. Wie auch 2007 haben Sozialdemokraten und die DF überproportionale Anteile bei Wählern über 60 Jahren. Die beiden bürgerlichen Parteien V und DF schnitten zudem überdurchschnittlich gut in den ländlichen Gebieten Dänemarks ab, während die Großstädte und insbesondere Kopenhagen Hochburgen der Parteien des „roten Blocks“ (S, SF, RV und EL) blieben.

\section{Regierungs- und Oppositionsbildung}

Die Regierungsbildung verläuft in Dänemark nach den Grundsätzen des negativen Parlamentarismus: Einzige formelle Bedingung ist der Nachweis, dass keine parlamentarische Mehrheit gegen die Regierungskonstellation eines designierten Ministerpräsidenten besteht. Dies wird durch Konsultation der Parteivorsitzenden bei der Königin festgestellt (so genannte „Königinnenrunde“). 34

Nach zweieinhalbwöchigen Sondierungen und Koalitionsverhandlungen stellte die neue Ministerpräsidentin Helle Thorning-Schmidt am 3. Oktober ihr Regierungsprogramm und ihr dreiundzwanzigköpfiges Kabinett der Königin vor (vgl. Tabelle 3). Für Aufsehen sorgte zuvor der Rückzug des designierten Finanzministers Henrik Sass Larsen (S), der vom dänischen Geheimdienst als nicht vertrauenswürdig angesehen wurde, da Verbindungen ins Rockermilieu nachgewiesen werden konnten. ${ }^{35}$

Nach zähen Verhandlungen wurde eine Minderheitsregierung aus Sozialdemokraten, Sozialliberalen und Sozialisten gebildet, welche von der Einheitsliste toleriert wird. Die Sozialistische Volkspartei ist somit zum ersten Mal in ihrer 52-jährigen Geschichte an einer Regierung beteiligt. Die so genannte Regierungsgrundlage, die in etwa einem Koalitionsvertrag entspricht, trägt dabei die deutliche Handschrift der Radikalen, die viele ihrer zentralen Wahlkampfforderungen durchsetzen konnte, während Sozialdemokraten und Sozia-

31 Schmidt Nielsen erhielt 47.002 persönliche Stimmen im Wahlkreis Groß-Kopenhagen und lediglich der amtierende Ministerpräsident Løkke Rasmussen erzielte mit 56.285 Stimmen mehr persönliche Stimmen.

32 Vgl. Jesper Eising, Thomas Larsen: Johanne fik et Fribillet, in: Berlingske Tidende - Politiko, http://www.b.dk/politiko/thomas-larsen-johanne-fik-en-fribillet (Abruf am 4. Oktober 2011).

33 Vgl. Søren Risbjerg Thomsen, a.a.O. (Fn. 24).

34 Für detailliertere Beschreibungen vgl. Erik Damgaard, Denmark: The Life and Death of Government Coalitions, in: Wolfgang C. Müller / Kaare Strøm (Hrsg.), Coalition Governments in Western Europe, Oxford 2000, S. 231 - 263.

35 Vgl. Hans Davidsen-Nielsen / Rasmus Bech, Thorning var klar til at vrage Henrik Sass Larsen, in: Politikken vom 1. Oktober 2011. 


\begin{tabular}{|c|c|}
\hline Ministerpräsidentin & Helle Thorning-Schmidt $(\mathrm{S})$ \\
\hline Wirtschaft und Inneres & Margrethe Vestager (RV) \\
\hline Äußeres & Villy Søvndal (SF) \\
\hline Finanzen & Bjarne Corydon (S) \\
\hline Justiz & Morten Bødskov (S) \\
\hline Wissenschaft und Innovation & Morten Østergaard (RV) \\
\hline Steuern & Thor Möger Pedersen (SF) \\
\hline Transport & Henrik Dam Kristensen $(\mathrm{S})$ \\
\hline Industrie & Ole Sohn (SF) \\
\hline Wohnungsbau, Stadt-und Landentwicklung & Carsten Hansen (S) \\
\hline Arbeit & Mette Frederiksen $(\mathrm{S})$ \\
\hline Bildung & Christine Edda Antorini (S) \\
\hline Soziales und Integration & Karin Hakkerup (S) \\
\hline Ernährung, Landwirtschaft und Fischerei & Mette Gjerskov (S) \\
\hline Klima, Energie und Bau & Martin Lidegaard (RV) \\
\hline Handel & Pia Olsen Dyhr (SF) \\
\hline Gesundheit & Astrid Krag (SF) \\
\hline Verteidigung & Nick Hakkerup (S) \\
\hline Umwelt & Ida Auken (SF) \\
\hline Europa & Nicolai Wammen (S) \\
\hline $\begin{array}{l}\text { Gleichstellung, Kirche und nordische Zusammen- } \\
\text { arbeit }\end{array}$ & Manu Sareen (RV) \\
\hline Entwicklungshilfe & Christian Friis Bach (RV) \\
\hline Kultur & Uffe Elbak (RV) \\
\hline
\end{tabular}

listen viele Kompromisse eingehen mussten. ${ }^{36}$ Die Vorsitzende der Radikalen Margrethe Vestager wurde mit einem Superministerium für Wirtschaft und Inneres ausgestattet, das einen starken Einfluss ihrer Partei im Laufe der Wahlperiode sichern soll. Politische Kommentatoren und Boulevardmedien interpretierten die Regierungsgrundlage im Wesentlichen als Fortsetzung der Wirtschaftspolitik der bürgerlichen Vorgängerregierung und als Bruch von Wahlkampfversprechen durch S und Sozialisten. ${ }^{37}$

So wurden die von Sozialdemokraten und Sozialisten geforderte Besteuerung hoher Einkommen und eine gesonderte Bankensteuer von den Sozialliberalen abgelehnt und im Gegenzug sogar eine langfristige Senkung der Einkommensteuer in Aussicht gestellt. Die Regierung verpflichtete sich zudem, die die im Frühjahr beschlossene Rentenreform trotz der Wahlkampfversprechen von S, SF und EL umzusetzen und sowie die Halbierung der Bezugszeit des Arbeitslosengeldes beizubehalten. Lediglich bei der Zuwanderungspolitik mussten die Radikalen einige Kompromisse mit $S$ und SF eingehen, da diverse restriktive Regelungen der bürgerlichen Regierung zunächst beibehalten werden sollen. Dies dürfte

36 Vgl. Christian Hüttemeier / Dorte Ipsen Boddum / Knud Brix, De Radikale sætter sig på Økonomien, in: Jyllands-Posten vom 4. Oktober 2010.

37 Vgl. Ralf Pittelkow, De Radikale minus 20 pct, in: JP.dk vom 3. Oktober 2011, http://jp.dk/ opinion/pittelkow/article2565429.ece (Abruf am 4. Oktober 2011); Thomas Larsen, Thornings kovending, in: Berlingske Tidende vom 6. Oktober 2011. 
künftig für interne Auseinandersetzungen bei Sozialdemokraten und Sozialisten sorgen, die sich bereits in den Tagen nach Bekanntgabe der Regierungsgrundlage und des Kabinetts abzeichneten. ${ }^{38}$ Die Ernennung des SF-Vorsitzenden Villy Søvndal zum Außenminister könnte sich durch dessen häufige Abwesenheit bei schwieriger innenpolitischer Lage als Problem erweisen, auch wenn dem durch Übertragung einiger Kompetenzen an andere Ministerien Rechnung getragen wurde.

Weitere Spannungen dürften zwischen Sozialliberalen und der Einheitsliste im Bereich der Sozial- und Wirtschaftspolitik zu erwarten sein. Während die Radikalen sich offen für weitere Einsparungen und Sozialstaatsreformen zeigen, gibt sich die Einheitsliste kompromisslos bei Kürzungen und als Sparbestrebungen aufgefassten Reformen. Eine mögliche Lösung, um den Gesichtsverlust beider Parteien zu vermeiden, wäre eine Enthaltung der Einheitsliste bei kritischen Abstimmungen. Hier könnte dann eine der bürgerlichen Parteien ein Mehrheitsbeschaffer sein, etwa die Konservativen.

Alle vier zukünftigen Oppositionsparteien hielten an ihren Vorsitzenden trotz des Verlustes der Regierungsmacht beziehungsweise des parlamentarischen Einflusses fest. Jedoch deutete sich ein Bruch ihrer bisher engen Zusammenarbeit gleich nach der Wahl an. ${ }^{39}$ Venstres Vorsitzender Lars Løkke Rasmussen teilte mit, dass seine Partei aufgrund des schwachen Abschneidens der Konservativen zukünftig nicht mehr zwangsläufig mit ihnen eine Koalition bilden wird. Eine ähnliche Reaktion zeigte die Vorsitzende der DF Pia Kjarsgaard, die eine Regierungsbeteiligung der Konservativen mit Unterstützung der DF in der Zukunft ebenso ausschloss. ${ }^{40}$ Deren Vorsitzender Lars Barfoed hatte zuvor bereits eine eigenständigere Oppositionsarbeit seiner Partei angekündigt. ${ }^{41}$ Das politische Überleben der Konservativen könnte sich als schwierig gestalten, sollte es weiterhin an zugkräftigen Themen sowie markanten Politikern mangeln und es so anderen bürgerlichen Parteien möglich werden, deren verbliebenes Wählersegment verstärkt zu umwerben.

Eine weitere offene Frage dürfte die Dauer der laufenden Wahlperiode betreffen. Die parlamentarische Situation könnte die neue Regierung dazu bewegen, bei günstigen Umfrageergebnissen auf Neuwahlen zu setzen, um nicht mehr zwangsläufig auf die Nordatlantischen Mandate oder die starke Einheitsliste angewiesen zu sein, und etwa die Konservativen für eine verstärkte Kooperation zu gewinnen, wie dies von Vestagers Radikalen gewünscht wird. Dies hängt jedoch vom zukünftigen Zuspruch für die Sozialdemokraten und die Sozialisten ab, deren Popularität aufgrund der gebrochenen Wahlversprechen weiter schwinden könnte. In diesem Fall dürften $S$ und SF kein Interesse an einer vorgezogenen Wahl haben. ${ }^{42}$

38 Vgl. Rasmus Bøttcher Christensen / Morten Skarbak, Frustration blandt vragede SF'ere, in: Politikken vom 4. Oktober 2011; Thomas Vibjerg / Knud Brix / Eva Aagaard / Dorte Ipsen Boddum / Christian Hüttemeier, Det buldrer og brager i det røde Bagland, in: Jyllands-Posten vom 5. Oktober.

39 Vgl. Knud Brix / Dorte Ipsen Boddum, Blå Blok opgiver fælles Opposition, in: Jyllands-Posten vom 6. Oktober 2011.

40 Siehe Bjarne Steensbeck / Annette Bonde / Uffe Tang, VK-alliancen er knækket, in: Berlingske Tidende vom 25. September 2011; Chris Kjer Jessen / Lena Masri, DF trækker Støtte til en Regering med de Konservative, in: Berlingske Tidende vom 26. September 2011.

41 Vgl. Annette Bonde / Uffe Tang, Barfoed afviser samlet Opposition, in: Berlingske Tidende vom 23. September 2011.

42 Vgl. Christine Cordsen, Der er lang Tid til næste Valg, in: Jyllands-Posten vom 7. Oktober 2011. 